stehen und insbesondere die Kranken ganz vorzüglich beköstigen. Die Wahl des Hauses hat sich also in jeder Hinsieht als glücklich erwiesen.

Dasselbe kann von den Einriclitungen gesagt werden, die zur Erreichung der ärztlichen Ziele getroffen und nunmehr zu einem ge wissen Abschluß gebracht worden sind. Thre Einführung und Weiterentwicklung wurde mir wesentlich erleichtert durch die Mitarbeit der Chefärzte Stabsarzt Dr. K rause (Neudorf) und seines Nachfolgers Stabsarzt Prof. Dr. Rosenfeld, der auch jetzt noch die Heilstätte leitet. Es handelte sich im wesentlichen um die weitcre Ausgestaltung der ,Heilbeschäftigung“", über welchc ich in einem früheren Aufsatzi ${ }^{1}$ be. richtet habe.

Schon vor der Eröffnung von Neuhof hatten wir in der Thomasschule, dem städtischen Lazarett, auch solche Beschäftigungszweigc gepflegt, die an die körperliche Leistungsfähigkeit größere Ansprüche stellen, also neben den Turnen die Schreinerei und auch die Feldarbeit. Diese konnte sich aber bei der großen Entfernung der Arbeitsplätze vom Lazarett nicht entwickeln. So gingen mit der Errichtung des Lazaretts Neuhof die "schweren" Spezialitäten naturgemäß auf dieses über. Damit war für die Thomasschule eine gewisse Entsagung verbunden, insofern fortan gerade ihre kräftigsten und leistungsfähigsten Insassen sobald als möglich nach Neuhof verlegt werden mußten. Dieser Gesichtspunkt konnte aber deshalb nicht ernsthaft in Betracht kommen, weil beide Lazarette von vornherein auf enges Zusammenarbeiten eingestellt und gerade auch in bezug auf die Beschäftigungstherapie (Heilbeschäftigung) nach einheitlichen Grundsätzen organisiert waren. Ich kann demnach im Folgenden über bcide Lazarette gemeinsam berichten.

\section{Weitere Erfahrungen mit der Heilbeschäftigung nervenkranker Soldaten.}

Von Prof. R. Wollenberg in Straßburg i. E., Chefarzt des Festungs-Lazaretts XXVII (Thomasschule) und des Hilfslazaretts XXVIIa (Nervenklinik), Fachärztlicher Beirat.

Die Erfahrungen im ersten Kriegsjahr veranlaßteu mich zu Anfang dieses Jahres, bei der zuständigen Stelle die Einrichtung einer Art ländlicher Militär-Nervenheilstätte anzuregen. Diese sollte geeigneten $\mathbf{K}$ ranken der Nerven-Lazarette Gelegenheit $\mathrm{zu} \cdot$ gesundheitsfördernder und nutzbringender Garten-, Feld- und Waldarbeit gewähren, zugleich aber den Aerzten, durch die Möglichkeit fortløufender Beobachtung und unmittelbarer Feststellung der tatsächlichen Leistungen der Kranken, für ihr Schlußgutachten einen zuverlässigeren Maßstab an die Hand geben, als ihn der gewöhnlich Lazarettaufenthalt gewährt. Die Heilstätte war also zumächst gedacht als eine Ergänzung des in der Stadt vorhandenen Hauptlazaretts und sollte im allgemeinen nur solche Kranke aufnelımen, welche durch jenes lindurchgegangen waren und die Wiederherstellung bis zu einem der drei Grade militärischer Verwendbarkeit mindestens nit Wahrscheinlicllkeit erwarten ließen.

Dieser Plan konnte durch das Entgegenkommen des Garnisonarztes, Generaloberarzt Dr. Hormanı, dadurch in befriedigender Weise verwirkliclit werden, daß die bisher für innerlich Kranke verwendete katholisclie Taubstummenanstalt für den gedachten Zweck zur Verfügung gestellt wurde.

Nachdem sich die Nervenheilstätte hier nunmehr seit $\Lambda \mathrm{n}$ fang Mai, also rund sechs Monate, in Betrieb befindet, ist es an der Zeit, über die bisherigen Erfahrungen und über einige Einzelheiten unserer Organisation zu berichten. Ich entspreche damit zugleich einem Wunsche, der mir voll auswärtigen Besuchern unserer Lazarette verschiedentlich ausgesprochen worden ist.

Die Taubstummenanstalt, ein stattliches Gebäude im Korridorstil, liegt, etwas abseits von den letzten Häusern des Straßburger Vorortes Neuhof, in ländlicher Umgebung, ist aber von der Stadtmitte aus mittels Straßenbahn in einer halben Stunde bequem zu erreichen. Das Haus eignet sich durch seine freie und aussichtsreiche Lage, durch seine Luft- und Lichtverhältnisse, die Art und Ausniessung der Räume und die vorzüglichen hygienischen Einrichtungen in hervorragender Weise für die Zwecke eines Lazaretts überhaupt, durch die reiche Arbeitsgelegenheit für die unsrigen im besonderen. Es besitzt neben geräumigen Werkstätten, die unter gewöhnlichen Verhältnissen den Zöglingen als Arbeitsräume dienen, einen Gemüse- und Obstgarten von etwa 1 ha Größe und ist in weitem Umkreis umgeben von Ländereien, welche die verschiedensten Feld- und Waldarbeiten gestatten. Dazu kommt als ein nicht zu unterschätzender Vorteil, daß die Schwestern des Hauses dem wirtschaftlichen Betrieb selbst mit besonderer Hingebung vor-

\section{Art und Verteilung der Arbeit.}

Die verschiedenen Handfertigkeiten werden als geeignete Beschäftigung für die schweren Kranken in beiden Lazaretten nach wie vor gepflegt; sie sind aber in mancher Hinsicht praktischer und auch mannigfaltiger geworden. In der gegenwärtigen Weihnachtszeit hat sich die Herstellung von Kinderspielzeug außerordentlich entwickelt, wobei aucl die Hausschreinerei gute Dienste leistet ${ }^{2}$.

Mit der Herausgabe des Materials und der ersten Anleitung bei der Arbeit sind mehrere Hilfsschwestern voll bescliäftigt. Dabei wird auf Anregungen, die von den Kranken selbst kommen, grundsätzlich so viel wie möglich eingegangen. Da in der Thmoasschule immer ziemlich viele schwerbewegliche Kranke vorhanden sind, wird dort auch in den Krankensälen selbst gearbeitet, natürlich nur insoweit dadurch keine Störungen entstehen.

In Neuhof ist ein besonderer Arbeitssaal eingerichtet, der unter der Leitung einer Schwester (Handarbeitslelireriu) steht. In einzelnen sind dort die verschiedenen Arbeitszweige und die Beteiligung der Kranken daran auf einer Uebersichtstafel zusammengestellt, die unmittelbar neben dem Hauseingang angebracht ist und täglich berichtigt wird. Wir sehen daraus, daß von dell Kranken des Lazaretts beispielsweise am 8. August 1915 bei einer Belegungszahl von 145 beschäftigt waren

$$
\begin{aligned}
& \text { mit Feldarbeit vormittags 49, nachmittags } 51 \text {; } \\
& \text { ", Gartenarbeit " } \quad 39, \quad \text {, } \quad 34 \text {; } \\
& \text { "Werkstättenarbeit } \quad, \quad 9, \quad, \quad 8 \text {, } \\
& \text { ", Handfertigkeiten ", 21, , } 21 .
\end{aligned}
$$

Der Rest ist als bettlägerig oder beurlaubt geführt.

Bei dem allmorgendlich stattfindenden Appell, bei dem sich alle nicht bettlägerigen Kranken einzufinden haben, werden dic Arbeiter verteilt, wobei, neben der allgemeinen persönlichen Eignung, der Grad der gegenwärtigen Leistungsfähigkeit maßgebend ist. Im allgemeinen wird ein Fortschreiten voll den Handfertigkeiten zu der Außenarbeit angestrebt wobei die Gartenarbeit den Uebergang bildet. Meist werden nur halbe Arbeitstage angenommen, derart, daß die Kranken nach ihrer Wahl entweder am Vormittag oder am Nachmittag je drei bis vier Stunden tätig sind.

Nach der Eröffnung von Neuhof fand ein großer Teil der Kranken sofort zweckentsprechende Beschäftigung in den Gemüsegärten der Anstalt, die seither vollständig durch

1) D. m. W. 1915 Nr. 26. $-{ }^{2}$ ) Es ist bedauerlich, daß die oft recht eigenartigen und zum Teil auch vom kunstgewerblichen Standpunkt aus leineswegs wertlosen Frzeugnisse der Lazarettbeschäftigung. wie sie gegenwärtig nicht nur hier, sondern an den verschiedengung: wie sie gegenwärtig nicht nur hier, sond irgend eincr: Stelle ge-
sten Orteil Deutschlands entstehen, nicht an irgend sammelt und für spätere Zeiten aufbewahrt werden 
Kranke besorgt worden sind. Dagegen schien es anfangs an sonstiger Gelegenheit zur Außenarbeit zu fehlen. Das Lazarett erbot sich deshalb der Stadt gegenüber, um erst einmal seine Leistungsfähigkeit zu beweisen, die Parkanlagen des in der Nähe gelegenen städtischen Gutes ,Wilhelmshof" in Stand zu setzen, und besorgte dies in den nächsten Wochen mit bestem Erfolg. Bald begannen dann die Landwirte und Unternehmer der Umgegend, sich um Arbeitskräfte zu bemühen. Die ZahI der so beschäftigten Kranken ist aus der unten mitgeteilten Tabelle zu ersehen: sie betrug in den letzten Monaten mehr als $50 \%$ der Lazarettinsassen. Die Kranken waren zuletzt auf 26 Arbeitsstellen verteilt und wurden hier mit ländlichen Arbeiten, wie Jäten, Mähen, Heumachen, auch mit Führen von Gespannen, Erdarbeiten etc. beschäftigt. Bei der Verteilung wird seitens der Aerzte auf die Art der Kranken auch insofern Rücksicht genommen, als gesundheitliclı unsichere Leute in möglichster Nähe des Lazaretts beschäftigt und mit ganz Zuverlässsigen in einer Gruppe vereinigt werden. Die Kontrolle der Arbeitergruppen erfolgt teils durch Aerzte des Lazaretts, teils durch einen landwirtschaftlich erfahrenen und ortskundigen Lazarettangestellten, welcher selbst aus den Reihen der Kranken hervorgegangen ist. Dieser nimmt auch die Wünsche der Arbeitgeber wegen Ueberweisung von Leuten entgegen und vermittelt das Geschäftliche (s. u.). Einige Male wurden auch Facharbeiter in öffentlichen und privaten Werkstätten, als Schlosser, Sattler, Eis dreher etc. aushilfsweise beschäftigt. Auch bei den Kranken, welche vor- und naclmittags arbeiten - was zur Erntezeit häufiger vorkam wurde mit seltenen Ausnahmen grundsätzlich daran festgehalten, daß sie nicht längere Zeit vom Lazarett fortblieben, insbesondere das Mittagessen in Lazarett nahmen und des Abends spätestens um $7 \frac{1}{2}$ Uhr heimkamen. Dies entsprach auch den Wünschen der Bauern, welche lieber etwas mehr zahlten, als die Leute in Kost nahmen. Alkoholgenu $B$ ist streng untersagt. Vom Lazarett selbst wird eine Kontrolle durch Nummern ausgeübt, welche, im Polizeizinımer des Lazaretts aufgehängt, beim Verlassen des Hauses initzunehmen, bei der Rückkehr'wieder an ilıren Platz zu bringen sind.

\section{Gewinnung der Mittel zur Beschaffung des Arbeitsmaterials.}

Die Lazarette sind in dieser Hinsicht fast ausschließlich auf sich selbst angewiesen. Die Thomasschule erwarb sich zunächst ein kleines Kapital durch eine Lotterie, zu welcher hiesige Künstler, wie Seebach, Daubner, Jäger, Schnug und Leschhorn, wertvolle Werke geschenkt hatten. Später fanden in beiden Lazaretten mehrfach Ausstellungen mit Verkauf von Arbeiten der. Kranken statt. Die auf diesem Wege gewonnenen, nicht unerheblichen Summen reichen aber, obwohl dazu noch kleinere Zuschüsse aus Mitteln des Roten Kreuzes kamen, nicht aus, um die Materialkosten zu decken. Dies geht daraus hervor, daß z. B. für die Thomasschule die monatlichen Ausgaben des Lazaretts für Beschäftigungszwecke in der letzten Zeit nahe an $1000 \mathrm{M}$ herankamen. Ein Teil der Summe kommt dadurch wieder ein, daß den Kranken, die ihre Arbeiten für sich oder ihre Angehörigen zu behalten wünschen, das Material zum Selbstkostenpreis angerechnet wird, daß also das Lazarett nur die Materialien für solche Arbeiten bezahlt, die in seinem Besitz verbleiben und bei Ausstellungen zu seinem Nutzen verkauft werdell. Barvergütungen werden vom Lazarett für die Arbeit nicht gezahlt, woll aber gelegentliche Zulagen in Gestalt von Tabak, und bei besonderen Anlässen auch wohl kleine Geldgeschenke ge währt.

Das Lazarett Neuhof verfährt mit Bezug auf die Hausarbeiten ebenso. Auch für die Gartenarbeiten wird dort keine Vergütung in bar, sondern nur in Naturalien, wie Limonade, Wurst etc. gewährt. Durch seine besonderen Arbeitszweige außerhalb des Hauses nimmt Neuhof aber eine bevorıugte Stelle ein. Denn diese sind nicht nur für die Kranken, sondern auch für das Lazarett selbst mit nicht geringen Einkünften verbunden. Die Landwirte und sonstigen Unternehmer zallen nüunlich an das Lazarett pro Arbeiter und vollen Arbeitstag $1,50 \mathrm{M}$, hiervon fallen $80 \mathrm{Pf}$. für den ganzen oder $40 \mathrm{Pf}$. für den halben Arbeitstag den betreffenden Kranken zu. Der Rest fließt in eine besondere Arbeitskasse des Lazaretts, über deren Zweck und Verwaltung unten noch das Notwendige ge- sagt werden wird. Diese Einrichtung, welche ich für sehr wichtig halte, ist deshalb getreffen, damit die Kranken sich bewußt bleiben, daß die Arbeit im Lazarett nicht dazu dienen soll, ihnen einen Gelderwerb zu verschaffen, sondern sie rascher gesund und wieder dienstfähig zu machen. Damit sie also die Arbeit niemals als' Selbstzweck, sondern immer nur als einen Faktor in der ärztlichen Behandlung ansehen, erhalten sie die obige Summe ausdrücklich nicht als Árbeitslohn, sondern als Arbeitsprämie und nicht direkt vom Arbeitgeber, sondern vom Lazarett. Gelegentliche Zeichen der Unzufriedenheit, die im Anfang dieser Einrichtung gegenüber laut wurden, sind verstummt, seitdem in einem besonderen Statut die Art der Verwendung dieser Arbeitskasse festgelegt und ein Vertreter der Kranken selbst zu ihrer Verwaltung mit herangezogen worden ist. Folgende Tabelle wird das Gesagtedeutlicher machen.

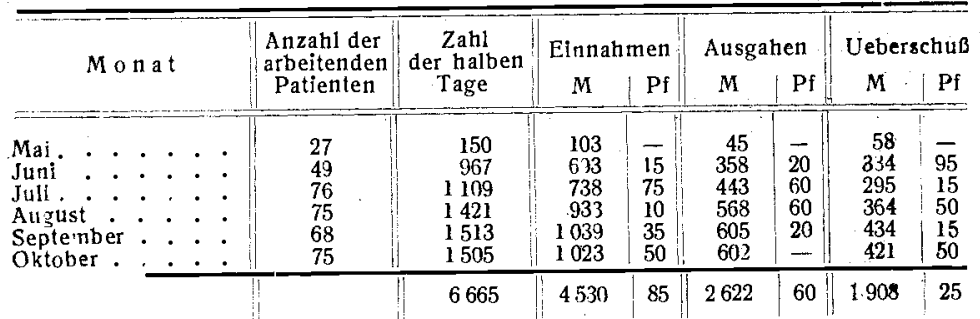

III. Eriahrungen bai der Heilbeschäftigung.

Ich sehe davon ab, hier Zahlen zu geben. Es genügt, darauf hinzuweisen, da $B$ in beiden Lazaretten die Beschäftigungslosigkeit zu den Ausnahmen gehört und daß fast jeder Insasse, der nicht durch seinen Krankheitszustand daran gehindert wird, sich irgendwie nützlich macht. Die so geschaffene Tradition wirkt auch auf die neuen Ankönmmlinge günstig ein, sodaß diese sich meist willig dem Geist des Hauses anpassen. Für die Güte der Løistungen unserer Außenarbeiter, die ja auch volkswirtschaftlich von unzweifelhaftem Wert sind, spricht der Umstand deutlich genug, daß die Insassen des Lazaretts Neuhof recht begehrte Arbeitskräfte geworden sind und daß ihnen von den Arbeitgebern fast durchweg ein sehr gïnstiges Zeugnis gegeben wird.

Auch vom ärztlichen Standpunkt aus kann man mit dem Erfolg wohl zufrieden sein, nicht nur wegen der Zahl der wieder verwendungsfähig Gewordenen, sondern auch wegen des offensichtlich günstigen Einflusses, den insbesondere der reichliche Aufenthalt im Freien und die nützliche Beschäftigung als solche auf unsere Nervösen ausübt. Auffallend selten ist es vorgekommen, daß Kranke wegen ihres Zustandes die angefangene Arbeit aufgeben mußten. Unterbrechungen durch Anfälle waren natürlich nicht seiten, hatten aber fast niemals eine ernstere Bedeutung.

Sehr wesentlich war endlich auch die Förderung, welche die Beurteilung der tatsächlichen Leistungsfähigkeit unserer Kranken durch die bei der Arbeit gemachten praktischen Beobashtungen erfahren hat. Um die so gewonnenen Anhaltspunkte auch für die zuküntige Nachuntersuchung möglichst festzulegen, sind besondere Schemata eingeführt, in welchen für jeden Fall auf Grund der Arbeitskontrolle die tatsächlichen Leistungen zur Zeit des Lazarettaufenthaltes und bei der Entlassung fortlaufend eingetragen werden. 